Check for updates

Cite this: RSC Adv., 2018, 8, 42322

\title{
A cubic room temperature polymorph of thermoelectric TAGS-85 $\uparrow$
}

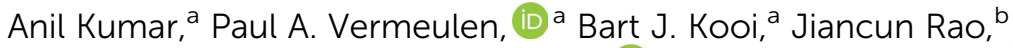 \\ Stefan Schwarzmüller, ${ }^{c}$ Oliver Oeckler ${ }^{(D)}{ }^{c}$ and Graeme R. Blake (D)*a
}

\begin{abstract}
The alloy $(\mathrm{GeTe})_{85}(\mathrm{AgSbTe})_{15}$, commonly known as TAGS-85, is one of the best performing p-type thermoelectric materials in the temperature range $200-500{ }^{\circ} \mathrm{C}$. In all reports thus far, TAGS- 85 adopts a rhombohedral crystal structure at room temperature and undergoes a reversible transition to a cubic phase in the middle of the operating temperature range. Here, we report on a novel, metrically cubic polymorph of TAGS-85 that can be obtained at room temperature using a particular cooling protocol during initial synthesis. This polymorph transforms irreversibly on initial heating to a 21-layer trigonal structure containing ordered cation vacancy layers, driven by the spontaneous precipitation of argyrodite-type $\mathrm{Ag}_{8} \mathrm{GeTe}_{6}$. We show that the precipitation of $\mathrm{Ag}_{8} \mathrm{GeTe}_{6}$ is detrimental to the thermoelectric performance of TAGS- 85 due to an increase in the vacancy concentration, which makes the samples more metallic in character and significantly reduces the Seebeck coefficient. The precipitation of $\mathrm{Ag}_{8} \mathrm{GeTe}_{6}$ can be suppressed by careful control of the synthesis conditions.
\end{abstract}

Received 6th July 2018

Accepted 27th November 2018

DOI: $10.1039 / \mathrm{c} 8 \mathrm{ra0} 5768 \mathrm{k}$

rsc.li/rsc-advances
Here we focus on the well-known solid solution (GeTe) $)_{x}$ $\left(\mathrm{AgSbTe}_{2}\right)_{100-x}$, which is commonly referred to as TAGS- $x$. The TAGS-85 composition has reported ZT values in the range 1.21.4 at $500{ }^{\circ} \mathrm{C}$, which is $\sim 30 \%$ lower than TAGS-80 at the same temperature. ${ }^{9}$ However, TAGS-85 is mechanically more stable ${ }^{\mathbf{1 0}}$ and is thus generally preferred for applications. TAGS- $x$ exhibit inherently complex nanostructures involving compositional and structural modulations, the spontaneous formation of nano-precipitates, twin and anti-phase domain boundaries, which all contribute to a low lattice thermal conductivity. ${ }^{11-13}$

In the literature, TAGS-85 ceramics have been prepared and processed by different methods. In some cases ingots are obtained by direct quenching from the melt ${ }^{\mathbf{1 4 , 1 5}}$ or held first at an intermediate temperature, ${ }^{\mathbf{1 1}, \mathbf{1 2}}$ and in other reports additional processing steps are carried out on as-prepared ingots such as subsequent annealing ${ }^{10}$ or grinding and hot-pressing..$^{\mathbf{9 1 6 , 1 7}}$ Both from our own work $^{\mathbf{1 8}}$ and from other previous reports, ${ }^{\mathbf{9}, \mathbf{1 3 - 1 5}}$ there are suggestions that TAGS-85 samples are often inhomogeneous or multi-phase in nature as evidenced by irregular or asymmetric peak shapes in X-ray diffraction measurements. This has motivated us to look more closely at whether the crystal structure and homogeneity can be influenced by careful control of the chemical synthesis conditions. We recently reported on the relationship between the structural and thermoelectric properties of TAGS-85 samples stabilized as a single-phase rhombohedral $\mathrm{R3} \mathrm{m}$ structure at room temperature. ${ }^{18}$ Contrary to earlier reports of a direct phase transition to a face-centered cubic $(F m \overline{3} m)$ phase above $380{ }^{\circ} \mathrm{C}$, we showed that the transformation proceeds via an intermediate trigonal phase containing layers of ordered cation vacancies. In terms of electronic
${ }^{a}$ Zernike Institute for Advanced Materials, University of Groningen, Nijenborgh 4, 9747 AG Groningen, The Netherlands. E-mail: g.r.blake@rug.nl

${ }^{b}$ AIM Lab, Maryland NanoCenter, University of Maryland, College Park, Maryland 20742, USA

${ }^{\prime}$ Institute for Mineralogy, Crystallography and Materials Science, Leipzig University, Scharnhorststraße 20, 04275 Leipzig, Germany

$\dagger$ Electronic supplementary information (ESI) available: Chemical composition analysis; XRD patterns of samples obtained using different cooling procedures; electron diffraction patterns; TGA/DSC measurements. See DOI: 10.1039/c8ra05768k 
structure, it is thought that the high-temperature cubic phase of TAGS-85 and other GeTe-related materials exhibits a greater degree of valence band valley degeneracy than the rhombohedral phase, which might significantly enhance the Seebeck coefficient. ${ }^{19,20}$ Therefore, it is of interest to study whether the transition to the cubic phase of TAGS-85 can be lowered in temperature. Here we report that a metastable cubic phase can indeed be stabilized at room temperature by careful adjustment of the synthesis conditions, but that it transforms irreversibly to a novel trigonal structure on initial heating. We study how the structural and thermoelectric properties of these phases evolve with repeated thermal cycling over the relevant operating temperature range.

\section{Experimental}

TAGS-85 samples were synthesized by reaction in evacuated sealed tubes. The elements Ge, Te, Ag and Sb (purity 99.99\%) were weighed in stoichiometric amounts, mixed using a mortar and pestle and placed in a quartz ampule, which was evacuated to $10^{-2}$ to $10^{-3}$ torr $(1.33-0.133 \mathrm{~Pa})$ using a rotary vane pump and then sealed using a flame; immediately before sealing the end of the ampule containing the reactants was cooled in liquid nitrogen. The ampule was heated in a tubular furnace at $850{ }^{\circ} \mathrm{C}$ for 1 hour to melt the reaction mixture, and the ampule was rotated every 10 minutes in order to ensure good homogeneity. Samples were then cooled to $500{ }^{\circ} \mathrm{C}$ over a period of $4 \mathrm{~h}$, at which temperature they were held for different lengths of time before quenching to room temperature in water. The samples were obtained in the form of shiny ingots with irregular, approximately rectangular shapes. For physical property measurements, the ingots were sliced to appropriate dimensions using a diamond wire saw and then polished to obtain flat surfaces and uniform thickness.

X-ray powder diffraction (XRD) patterns were recorded on crushed ingots using a Bruker D8 diffractometer operating in Bragg-Brentano geometry with $\mathrm{Cu} \mathrm{K} \alpha_{1}$ radiation and combined with an Anton Paar TTK-450 hot stage. The sample chamber was evacuated to $\sim 10^{-3}$ mbar $(\sim 0.1 \mathrm{~Pa})$ prior to the heating stage being switched on. Temperature was varied using a TCU-100 control unit, which has a precision of within $\pm 1{ }^{\circ} \mathrm{C}$. Heating and cooling rates of $0.5{ }^{\circ} \mathrm{C} \mathrm{s}^{-1}$ between set-point temperatures were used; before beginning a measurement the sample was held for $300 \mathrm{~s}$ in order to ensure thermal equilibrium. A Huber G670 diffractometer operating in Guinier geometry with $\mathrm{Cu} \mathrm{K} \alpha_{1}$ radiation and combined with a closed-cycle refrigerator was used to obtain XRD data below room temperature. All data were analyzed using the GSAS software. ${ }^{21}$

Differential scanning calorimetry (DSC) and thermogravimetric analysis (TGA) measurements were performed using a TA-instruments STD 2960. A Pt crucible was used to measure ground up pieces of ingot over a temperature range of $30^{\circ} \mathrm{C}$ to $600{ }^{\circ} \mathrm{C}$ at a rate of $1 \mathrm{~K} \mathrm{~min}^{-1}$ under a flow of argon.

Seebeck coefficients and electrical conductivity were measured simultaneously using a Linseis LSR-3 apparatus, which utilizes a dc four-probe method. Thermal diffusivity $\left(D_{\mathrm{t}}\right)$ was measured by the laser flash method using a Linseis
LFA1000 apparatus equipped with an InSb detector; samples were measured in a He atmosphere. Up to five data points of $D_{\mathrm{t}}$ were merged after evaluating the quality of the fitted model ${ }^{22}$ and excluding outliers at each temperature step of $50{ }^{\circ} \mathrm{C}$, starting from $50{ }^{\circ} \mathrm{C}$ up to $450{ }^{\circ} \mathrm{C}$. Thermal conductivity was calculated using the formula $\kappa=D_{\mathrm{t}} \times d \times C_{\mathrm{p}}$. Here the density $d$ was calculated from the mass and volume of the sample determined by the Archimedes principle. The specific heat capacity $C_{\mathrm{p}}$ was obtained using the Dulong-Petit approximation, which has previously been shown to be valid for GeTebased materials given the large experimental uncertainties involved in measurements of $C_{\mathrm{p}}$. $^{\mathbf{9 1 9 , 2 3 , 2 4}}$ We estimate that the ZT values obtained by combining the above measurements exhibit an uncertainty of $\sim 20 \%$.

For TEM measurements, samples were sliced and glued inside brass tubes of $3 \mathrm{~mm}$ diameter and cut into disks. The disks were then ground, dimpled, and ion milled using a Gatan PIPS II at $6^{\circ}$ with an accelerating voltage that was ramped from 4 $\mathrm{kV}$ to $0.2 \mathrm{kV}$ to achieve electron transparency. TEM images and electron diffraction patterns were obtained using JEM 2010 and JEM $2010 \mathrm{~F}$ microscopes operated at $200 \mathrm{kV}$. EDS measurements were performed in the TEM, using a $\mathrm{Si}(\mathrm{Li})$ detector. Cliff-Lorimer fitting without absorbance was performed using the NSS 2.3 software (Thermo Scientific) to obtain accurate composition information. In some cases, samples were prepared by a FIB (focused ion beam) technique. TEM images and electron diffraction patterns were then obtained with a Tecnai G2 F30 STwin at an accelerating voltage of $300 \mathrm{kV}$.

\section{Results and discussion}

We have previously reported that the procedure used to cool samples of TAGS-85 from the melt has a strong influence on the homogeneity of the phase that is obtained at room temperature.$^{18}$ Single-phase samples can only be obtained by holding at an intermediate temperature; $>3 \mathrm{~h}$ at $600{ }^{\circ} \mathrm{C}$ gives a pure rhombohedral phase whereas shorter holding times give multiphase mixtures of rhombohedral phases with slightly different lattice parameters, implying varying chemical compositions. Here we show that a novel cubic polymorph of TAGS-85 can be obtained when the intermediate holding temperature during the initial cooling procedure is lowered to $500{ }^{\circ} \mathrm{C}$.

This cubic room temperature phase, which we refer to as the $\mathrm{C}_{\mathrm{RT}}$ phase, can be consistently obtained by holding the molten precursor at $850^{\circ} \mathrm{C}$ for $1 \mathrm{~h}$, cooling to $500^{\circ} \mathrm{C}$ over $4 \mathrm{~h}$, holding at this temperature for $3 \mathrm{~h}$ and then quenching in water. The cubic symmetry is evidenced by the single $220 \mathrm{XRD}$ reflection shown in Fig. 1b. Longer holding times at $500{ }^{\circ} \mathrm{C}$ led to stabilization of a single rhombohedral phase at room temperature, evidenced by the splitting of the 220 peak into a doublet indexed as 211 and $10 \overline{1}$ in the rhombohedral setting (ESI, Fig. S1 $\dagger$ ), which suggests that the $\mathrm{C}_{\mathrm{RT}}$ phase is metastable. Direct quenching from the melt yielded a doublet of broad peaks (Fig. S1 $\dagger$ ), indicating an inhomogeneous rhombohedral sample. The chemical composition of the as-synthesized $\mathrm{C}_{\mathrm{RT}}$ sample was determined by EDX analysis performed at ten locations on the sample surface. The averaged chemical composition is listed in 


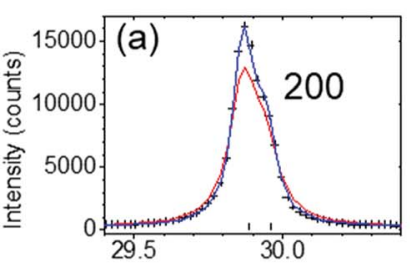

$1 \mu \mathrm{m}$

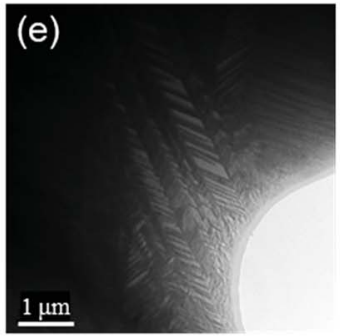

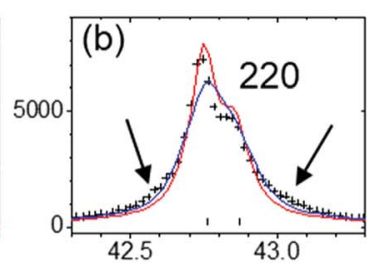

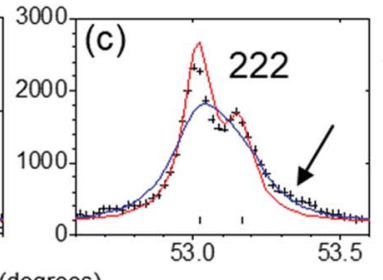

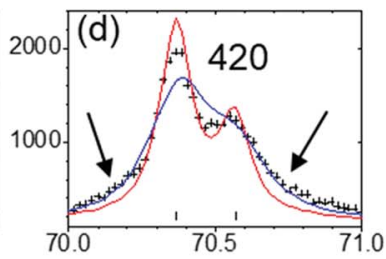

$2 \theta$ (degrees)
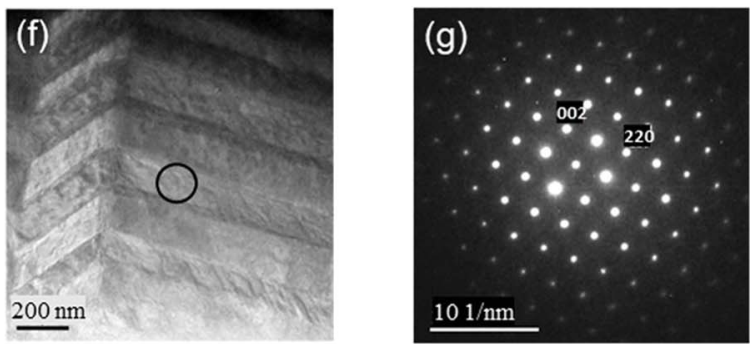

Fig. 1 Structural properties of $C_{R T}$ phase of TAGS-85. (a)-(d) Selected XRD peaks of as-synthesized $C_{R T}$ phase fitted without (red curve) and with (blue curve) the anisotropic microstrain broadening model. The arrows indicate broadening at the bases of peaks. All peaks appear as doublets due to $K \alpha_{1}$ and $K \alpha_{2}$ components of the X-ray beam. (e) and (f) Bright field TEM images for as-synthesized $C_{R T}$ phase. (g) [1110] zone axis SAED pattern obtained from the single-domain area marked by the black circle in (f).

Table S1 of the ESI $\dagger$ and does not deviate significantly from the nominal stoichiometry of TAGS-85.

At room temperature all XRD peaks of the $\mathrm{C}_{\mathrm{RT}}$ phase were indexed in the cubic $F m \overline{3} m$ space group. The pattern could be fitted using a rock salt-type structure model in which $\mathrm{Ge}, \mathrm{Ag}$ and Sb occupy the 4 a Wyckoff position with coordinates $(0,0,0)$ and Te occupies the $4 \mathrm{~b}$ position with coordinates $(0.5,0.5,0.5)$. In order to check whether a transition to the R-phase takes place below room temperature, we performed short XRD scans down to $20 \mathrm{~K}$. The 220 peak did not split, indicating that the unit cell stays metrically cubic. In fitting the room temperature data the peak shapes were modeled using a pseudo-Voigt function; however, close inspection revealed a considerable degree of anisotropic broadening, particularly at the bases of some reflections such as 220, 222 and 420 as shown in Fig. 1b-d. The sharpest peaks are 200 (Fig. 1a) and 400 (not shown). The Stephens anisotropic microstrain broadening model ${ }^{25}$ improved the fitting significantly but was unable to perfectly model those peaks exhibiting broadened bases. The refined crystallographic data for the $\mathrm{C}_{\mathrm{RT}}$ phase at room temperature are summarized in Table 1 and the fitted XRD profile is shown in the ESI, Fig. S2. $\dagger$

The relatively sharp 200 and 400 XRD peaks suggest longer structural coherence along $\{100\}$ than along other crystal

Table 1 Crystallographic data for Rietveld refinement of $C_{R T}$ phase of TAGS- 85 at $295 \mathrm{~K}$

Space group (no.)

Formula weight $\left(\mathrm{g} \mathrm{mol}^{-1}\right)$

Lattice parameter $(\AA)$

Cell volume $\left(\AA^{3}\right)$

$Z$ (formula units per cell)

X-ray density $\left(\mathrm{g} \mathrm{cm}^{-3}\right)$

Stephens anisotropic peak broadening parameters

$\mathrm{w} R_{\mathrm{p}}$

$\chi^{2}$ directions. This is consistent with the bright-field TEM images in Fig. 1e and $f$, which reveal a herringbone domain structure. Similar domains have been observed in GeTe, where the stripes tend to be oriented along $\{100\} .^{8}$ However, because such domains are only expected for the rhombohedral, polar phase of GeTe-related materials, it is likely that the $\mathrm{C}_{\mathrm{RT}}$ phase is only metrically cubic and that the true symmetry is lower on local length-scales, which diffraction techniques would be unable to probe directly. Fig. $1 \mathrm{~g}$ shows a [11̄0] zone axis SAED pattern obtained from the single domain marked by the black circle in Fig. 1f. The measured angle between the cubic (002) and (220) planes is $90.8^{\circ}$, consistent with a slight rhombohedral distortion. Further SAED patterns of the $\mathrm{C}_{\mathrm{RT}}$ phase are presented and discussed in the ESI, Fig. S3, $\uparrow$ but the results are inconclusive regarding symmetry. We note that similar metrically cubic phases have previously been observed for $\mathrm{Ge}_{1-x} \mathrm{Sb}_{x} \mathrm{Te}$, where small rhombohedral domains are strained and unable to establish their rhombohedral metrics. ${ }^{20,26}$

\section{High temperature structural properties}

XRD data taken when the cubic $\mathrm{C}_{\mathrm{RT}}$ phase was heated (Fig. 2a) show the appearance of new peaks at $180{ }^{\circ} \mathrm{C}$ followed by disappearance of the $\mathrm{C}_{\mathrm{RT}}$ phase at $240{ }^{\circ} \mathrm{C}$. The new set of peaks could be indexed using a trigonal unit cell with $a=4.215$ (3) $\AA, c$ $=38.27(7) \AA$ at $240{ }^{\circ} \mathrm{C}$. The $c$-axis of the trigonal structure corresponds to 21 alternating $\mathrm{Te}$ and $\mathrm{Ge} / \mathrm{Ag} / \mathrm{Sb}$ layers; we will refer to this structure hereafter as 21P following the Ramsdell notation, ${ }^{27}$ where $\mathrm{P}$ indicates a primitive unit cell. Weak XRD peaks corresponding to $\mathrm{Ag}_{8} \mathrm{GeTe}_{6}$ appear as a second phase above $180^{\circ} \mathrm{C}$ and coexist with the $21 \mathrm{P}$ phase up to the highest temperature measured $\left(390{ }^{\circ} \mathrm{C}\right)$. The $21 \mathrm{P}$ phase does not transform back to $\mathrm{C}_{\mathrm{RT}}$ on subsequent cooling to room temperature, thus the $\mathrm{C}_{\mathrm{RT}}$ to $21 \mathrm{P}$ transition is irreversible. Following previous studies of TAGS and GeTe, we expect that another transition to a high-temperature cubic (C) phase might occur at 


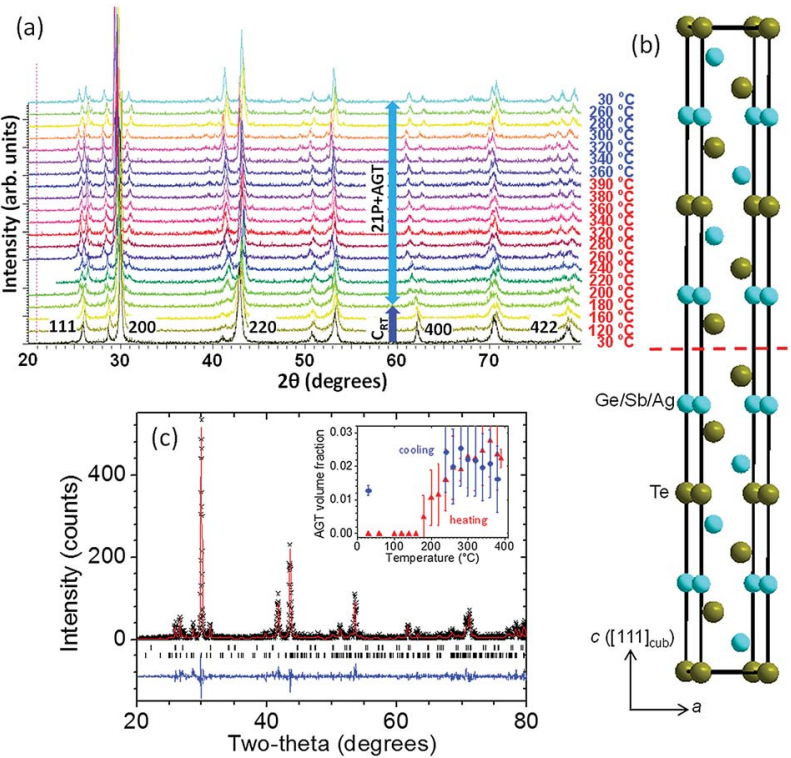

Fig. 2 High temperature structural properties of $C_{R T}$ phase of TAGS85. (a) XRD patterns collected on initial heating of as-synthesized $C_{R T}$ phase from $30^{\circ} \mathrm{C}$ to $390^{\circ} \mathrm{C}$ (bottom to top), followed by cooling back to $30^{\circ} \mathrm{C}$. (b) Schematic representation of one unit cell of $21 \mathrm{P}$ structure. The red dashed line indicates a layer of $\mathrm{Ge} / \mathrm{Sb} / \mathrm{Ag}$ vacancies. (c) Observed (black data points), calculated (red line) and difference (blue line) XRD profiles of 21P phase (lower tick marks) with $\mathrm{Ag}_{8} \mathrm{GeTe}_{6}$ impurity (upper tick marks) at $20^{\circ} \mathrm{C}$ after one heating + cooling cycle. The inset shows the evolution of the volume fraction of $\mathrm{Ag}_{8} \mathrm{GeTe}_{6}$ on initial heating (red) and subsequent cooling (blue).

temperatures above those accessed in our XRD measurements. Therefore, a DSC/TGA measurement was performed on heating from $30-600{ }^{\circ} \mathrm{C}$ under $\mathrm{Ar}$ atmosphere. The resulting curves are shown in the ESI, Fig. $\mathrm{S} 4, \dagger$ where a minimum in the DSC trace at $408{ }^{\circ} \mathrm{C}$ (endothermic) followed by a maximum centered at $449{ }^{\circ} \mathrm{C}$ (exothermic) is observed. We tentatively assign the endothermic peak to a $21 \mathrm{P} \rightarrow \mathrm{C}$ transition. The origin of the strong exothermic peak remains unclear. Increasing the temperature beyond $\sim 500{ }^{\circ} \mathrm{C}$ results in decomposition of the sample as shown by the onset of weight loss. A schematic diagram of the phase relations in these samples is shown in Fig. 3. The structure of the $21 \mathrm{P}$ phase was determined from data collected at $20^{\circ} \mathrm{C}$ after one heating and cooling cycle. An initial structural model was built in which there are 11 cation layers and 11 anion layers, using space group $P \overline{3} m 1$. While fitting the XRD pattern, the layers were initially spaced equally and a set of soft constraints was added to keep the $(\mathrm{Ge} / \mathrm{Ag} / \mathrm{Sb})$-Te distances close to $3.00 \AA$, the average value in the rhombohedral phase. Refinement of the $z$-coordinates of all atoms was then carried out. The precipitation of $\mathrm{Ag}_{8} \mathrm{GeTe}_{6}$ (refined phase fraction $\sim 2 \%$ by volume) suggests that the $21 \mathrm{P}$ phase might be more cation deficient than as-synthesized TAGS-85. Therefore, the possibility of a "vacant" cation layer at the $1 \mathrm{~b}(0,0,1 / 2)$ position was investigated and the fit significantly improved. The fitted XRD pattern measured at $20{ }^{\circ} \mathrm{C}$ is shown in Fig. $2 \mathrm{c}$ and a representation of the $21 \mathrm{P}$ structure is shown in Fig. 2b. The refined crystallographic data for $21 \mathrm{P}$ are listed in Table 2.

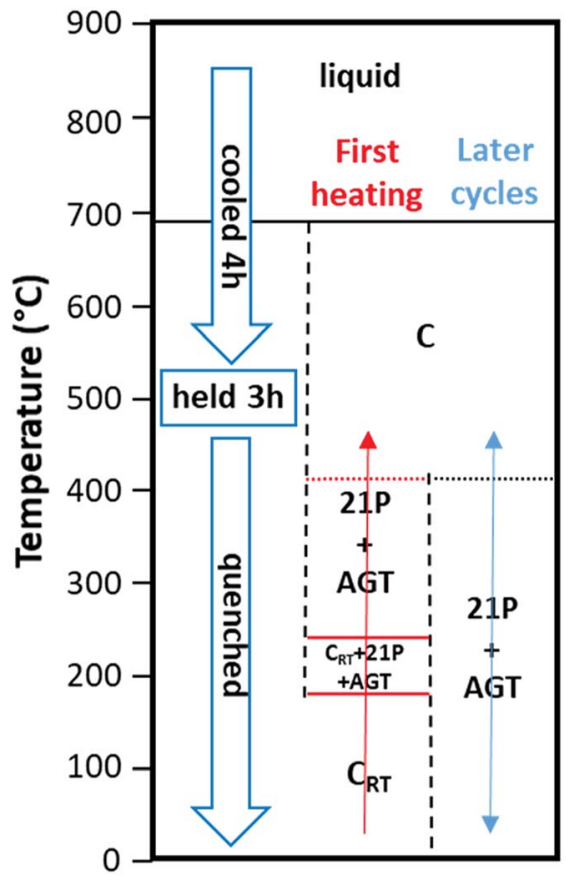

Fig. 3 Diagram showing phase transitions of $C_{R T}$ phase that occur on heating and subsequent thermal cycling. The labels 21P and AGT denote the 21-layer TAGS-85 polymorph (see main text for details) and an $\mathrm{Ag}_{8} \mathrm{GeTe}_{6}$ impurity phase, respectively. Solid horizontal lines indicate phase transition temperatures determined within an uncertainty of $\pm 10{ }^{\circ} \mathrm{C}$ and dotted horizontal lines indicate phase transition temperatures with a larger uncertainty than $\pm 10^{\circ} \mathrm{C}$ due to lack of data. These horizontal lines are color-coded: red = transition on initial heating only; black = transition on subsequent heating and cooling cycles.

Note that a metastable pseudo-cubic phase possibly similar to $\mathrm{C}_{\mathrm{RT}}$ has previously been reported for TAGS materials synthesized with intentionally large cation vacancy concentrations by varying the $\mathrm{Ag} / \mathrm{Sb}$ ratio. ${ }^{23}$ A similar irreversible phase transition was observed on initial heating, but to a 15-layer trigonal phase with considerable vacancy layer disorder; a further reversible transition to a cubic structure took place above $400{ }^{\circ} \mathrm{C}$. The as-synthesized pseudo-cubic phase was characterized by (111) vacancy layers that only extend over a few $\mathrm{nm}$ in the lattice and are not ordered in periodic fashion; they form "parquet like" multidomain nanostructures when observed by HRTEM. Heating this disordered cubic phase induces a vacancy diffusion process and transformation to a more thermodynamically stable trigonal phase in which the vacancies become ordered in layers in long-range periodic fashion, forming van der Waals gaps. Similar phases and transitions have been observed in other materials related to TAGS, for example (GeTe) ${ }_{n} \mathrm{Sb}_{2} \mathrm{Te}_{3}$ (commonly known as GST). ${ }^{28}$ For example, $\mathrm{Ge}_{2} \mathrm{Sb}_{2} \mathrm{Te}_{5}, \mathrm{Ge}_{1} \mathrm{Sb}_{4} \mathrm{Te}_{7}, \mathrm{Ge}_{1} \mathrm{Sb}_{2} \mathrm{Te}_{4}$ and $\mathrm{Ge}_{3} \mathrm{Sb}_{2} \mathrm{Te}_{6}$ adopt trigonal structures consisting of 9, 12, 21, and 33 alternating anion and cation layers, respectively. ${ }^{28-30}$ Analogous phase transitions have also been reported for GST materials doped with In, Co and Sn. ${ }^{31-33}$ Our TAGS-85 samples are nominally stoichiometric and charge-balanced. However, it is possible that TAGS samples contain intrinsically high defect 
Table 2 Refined structural parameters of $21 \mathrm{P}$ phase at $20{ }^{\circ} \mathrm{C}$ : space group $P \overline{3} m 1, a=4.1914(4) \AA, c=38.288(7) \AA ; w R_{p}=0.3407, \chi^{2}=1.357$

\begin{tabular}{llllll}
\hline Atom & Site & $X$ & $Y$ & $z$ & Site occupancy \\
\hline $\mathrm{Te}_{1}$ & $1 \mathrm{a}$ & 0 & 0 & 0 & 1 \\
$\mathrm{Ge}_{2} / \mathrm{Ag}_{2} / \mathrm{Sb}_{2}$ & $2 \mathrm{~d}$ & $1 / 3$ & $2 / 3$ & $0.0449(13)$ & $0.7391 / 0.1304 / 0.1304$ \\
$\mathrm{Te}_{3}$ & $2 \mathrm{~d}$ & $2 / 3$ & $1 / 3$ & $0.0910(7)$ & 1 \\
$\mathrm{Ge}_{4} / \mathrm{Ag}_{4} / \mathrm{Sb}_{4}$ & $2 \mathrm{c}$ & 0 & 0 & $0.1393(8)$ & $0.7391 / 0.1304 / 0.1304$ \\
$\mathrm{Te}_{5}$ & $2 \mathrm{~d}$ & $1 / 3$ & $2 / 3$ & $0.1824(8)$ & 1 \\
$\mathrm{Ge}_{6} / \mathrm{Ag}_{6} / \mathrm{Sb}_{6}$ & $2 \mathrm{~A}$ & $2 / 3$ & $1 / 3$ & $0.2314(9)$ & $0.7391 / 0.1304 / 0.1304$ \\
$\mathrm{Te}_{7}$ & $2 \mathrm{c}$ & 0 & 0 & $0.2778(5)$ & $0.043(5)$ \\
$\mathrm{Ge}_{8} / \mathrm{Ag}_{8} / \mathrm{Sb}_{8}$ & $2 \mathrm{~d}$ & $1 / 3$ & $2 / 3$ & $0.3245(9)$ & $0.069(5)$ \\
$\mathrm{Te}_{9}$ & $2 \mathrm{~d}$ & $2 / 3$ & $1 / 3$ & $0.3709(8)$ & 1 \\
$\mathrm{Ge}_{10} / \mathrm{Ag}_{10} / \mathrm{Sb}_{10}$ & $2 \mathrm{c}$ & 0 & 0 & $0.4219(8)$ & $0.043(5)$ \\
$\mathrm{Te}_{11}$ & $2 \mathrm{~d}$ & $1 / 3$ & $2 / 3$ & $0.4607(9)$ & $0.069(5)$ \\
& & & & & $0.043(5)$ \\
& & & & & $0.069(5)$ \\
\end{tabular}

concentrations that have a tendency toward ordering with heat treatment. We note that there are also several reports of localized structural distortions in rocksalt-type binary IV-VI semiconductors such as GeTe, SnTe, PbTe and PbS that appear cubic to diffraction techniques. ${ }^{34-39}$ Rather than involving short-range ordering of cation vacancies, the lowering of local symmetry in the "cubic" phases of these systems is thought to be associated with the relative displacement of the cation substructure with respect to the anion substructure along the cubic [111] direction (rhombohedral distortion). However, the concentration of cation vacancies might have a significant influence on the ordering length and magnitude of these ferroelectric-type displacements. ${ }^{39}$ It has also been reported in the case of GeTe that the cubic polymorph contains more cation vacancies than the rhombohedral polymorph. ${ }^{40}$ Anharmonic thermal vibrations further complicate the picture ${ }^{\mathbf{4 1 4 2}}$ and might falsely appear as atomic displacements to local structural probes such as pair distribution function analysis of diffraction data. ${ }^{43}$ Further work is thus required to better understand the relationship between cation vacancy ordering and the ordering of atomic displacements in IV-VI materials.

\section{Thermoelectric properties}

The thermoelectric properties of the $\mathrm{C}_{\mathrm{RT}}$ phase were measured over three thermal cycles between room temperature and $400{ }^{\circ} \mathrm{C}$ on slices from the same ingot with $90-93 \%$ of the density determined from XRD data, as shown in Fig. 4. Measurements on two different samples were averaged to obtain $\sigma, S$ and PF. Thermal conductivity was measured on two further samples and the data were also averaged. Averaging was performed because the measured properties on different samples were reproducible to within $\sim 5 \%(\sigma$ and $S)$ and $\sim 10 \%(\kappa)$ after the first heating cycle.

For the initial heating cycle only, the electrical conductivity varied greatly between the two samples. For one sample the electrical conductivity was initially only $\sim 1100 \mathrm{~S} \mathrm{~cm}^{-1}$ in the assynthesized $\mathrm{C}_{\mathrm{RT}}$ phase. A sudden increase to $\sim 1800 \mathrm{~S} \mathrm{~cm}^{-1}$ was observed at $\sim 180{ }^{\circ} \mathrm{C}$ where the irreversible transformation to the $21 \mathrm{P}$ phase occurs (inset to the plot of $\sigma$ in Fig. 4). In contrast, the initial electrical conductivity of the second sample was no different to that on subsequent thermal cycles. Therefore, averaging of the data was performed only from the first cooling cycle onwards. The main feature of the electrical conductivity plot is a broad maximum at $\sim 230{ }^{\circ} \mathrm{C}$ with no hysteresis. There is no evidence from XRD for any phase transition at this temperature after the initial heating cycle. However, the maximum temperature attainable of $390^{\circ} \mathrm{C}$ in our XRD measurements was insufficient to reach the expected transformation to the hightemperature cubic structure observed by DSC measurement. It is possible that this phase was reached during the electrical conductivity/Seebeck measurement (highest temperature 406 ${ }^{\circ} \mathrm{C}$ ), in which case the reverse $\mathrm{C} \rightarrow 21 \mathrm{P}$ transition might be significantly lowered on subsequent cooling.

The electrical conductivity is $\sim 40 \%$ higher than in our previously reported rhombohedral TAGS-85 samples ${ }^{\mathbf{1 8}}$ but the Seebeck coefficient is much lower, only reaching $100 \mu \mathrm{V} \mathrm{K}^{-1}$ at $400{ }^{\circ} \mathrm{C}$ and giving a lower power factor of $0.0016 \mathrm{~W} \mathrm{~m}^{-1} \mathrm{~K}^{-2}$. The high
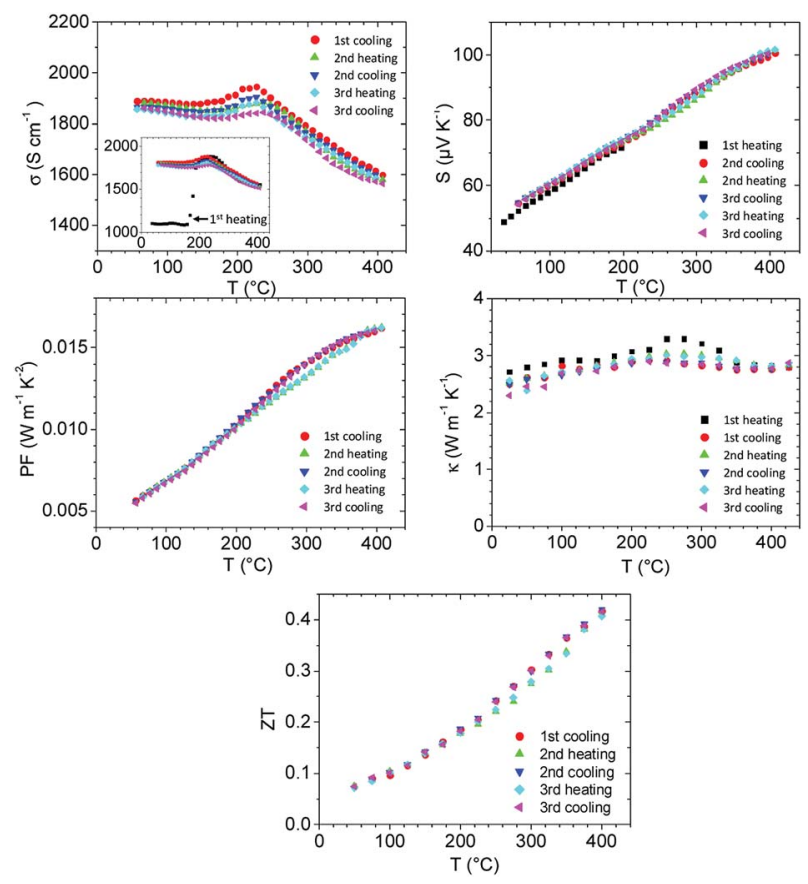

Fig. 4 Thermoelectric properties of $C_{R T} / 21 P$ phase of TAGS- 85 over three thermal cycles. The electrical conductivity $\sigma$, Seebeck coefficient $S$ and power factor PF are averaged from measurements on two samples, neglecting the initial heating cycle. The inset to the $\sigma$ plot shows data for the initial heating cycle of one sample. The thermal conductivity $\kappa$ was measured on two further samples and averaged. The figure of merit ZT was obtained by combining the averaged data presented here. 
electrical conductivity is reflected in a high thermal conductivity, which rises slightly from $\sim 2.5 \mathrm{~W} \mathrm{~m}^{-1} \mathrm{~K}^{-1}$ at $50^{\circ} \mathrm{C}$ to $\sim 2.9 \mathrm{~W} \mathrm{~m}^{-1}$ $\mathrm{K}^{-1}$ at $400^{\circ} \mathrm{C}$. Consequently, the maximum ZT is just over 0.4 at $400{ }^{\circ} \mathrm{C}$. The significantly worse thermoelectric properties of the $\mathrm{C}_{\mathrm{RT}} / 21 \mathrm{P}$ phase might be partially due to the higher volume fraction of the $\mathrm{Ag}_{8} \mathrm{GeTe}_{6}$ precipitate, which is $\sim 2 \%$ compared to $\sim 1 \%$ for our previous rhombohedral samples. ${ }^{18}$ This issue might also be linked to the different density of cation vacancy layers in the 21P structure of the current study and the 39-layer (39P) structure previously obtained on heating rhombohedral samples. The 21P structure has roughly double the density of vacancy layers, which might lead to a self-doping effect and a hole concentration that is higher than optimal with respect to thermoelectric performance. In an earlier study of $(\mathrm{GeTe})_{80}\left(\mathrm{Ag}_{y^{-}}\right.$ $\left.\mathrm{Sb}_{2-y} \mathrm{Te}_{3-y}\right)_{20}$ (TAGS-80) it was proposed that the poorer performance of silver-rich compositions could partly be attributed to a higher concentration of $\mathrm{Ag}_{8} \mathrm{GeTe}_{6}$ impurity. ${ }^{13}$ From our previous and current investigations it appears that the precipitation of $\mathrm{Ag}_{8} \mathrm{GeTe}_{6}$ in TAGS materials is spontaneous and therefore unavoidable. However, the concentration of the precipitate can be controlled by the initial synthesis conditions, which in turn appears to determine the density of cation vacancies. A larger concentration of vacancies leads to more metallic character and poorer thermoelectric performance.

\section{Conclusions}

We have obtained a novel metrically cubic $\left(\mathrm{C}_{\mathrm{RT}}\right)$ polymorph of TAGS-85 at room temperature by careful adjustment of the synthesis conditions. Peak width analysis of our XRD data and the observation of a complex domain structure by TEM suggest that the symmetry of the $\mathrm{C}_{\mathrm{RT}}$ phase might be lower on local length scales. This polymorph transforms irreversibly on heating to a new trigonal (21P) structure that contains ordered layers of cation vacancies. This occurs together with the spontaneous precipitation of $\sim 2 \% \mathrm{Ag}_{8} \mathrm{GeTe}_{6}$ by volume. The $\mathrm{C}_{\mathrm{RT}}$ polymorph and its associated trigonal phase exhibit higher electrical conductivity than the better studied rhombohedral polymorph but a much lower Seebeck coefficient and power factor, which is thus detrimental to the thermoelectric performance. The precipitation of cation-rich $\mathrm{Ag}_{8} \mathrm{GeTe}_{6}$ appears to occur more readily for the cubic polymorph, leaving additional cation vacancies. TAGS-85 thus becomes more metallic in character with inferior thermoelectric properties. Our study suggests that the best thermoelectric properties of TAGS- 85 are obtained when the spontaneous precipitation of $\mathrm{Ag}_{8} \mathrm{GeTe}_{6}$ is minimized, which can be achieved by careful control of the synthesis conditions.

\section{Conflicts of interest}

There are no conflicts to declare.

\section{Acknowledgements}

The authors thank J. Baas for technical support. This project is partially supported by the North Netherlands Partnership (SNN), Spatial Economic Programme.

\section{Notes and references}

1 G. J. Snyder and E. S. Toberer, Nat. Mater., 2008, 7, 105-114.

2 J. R. Sootsman, D. Y. Chung and M. G. Kanatzidis, Angew. Chem., 2009, 121, 8768-8792; Angew. Chem. Int. Ed., 2009, 48, 8616-8639.

3 M. G. Kanatzidis, Chem. Mater., 2010, 22, 648-659.

4 L.-D. Zhao, V. P. Dravid and M. G. Kanatzidis, Energy Environ. Sci., 2014, 7, 251-268.

5 S. Perumal, S. Roychowdhury and K. Biswas, J. Mater. Chem. C, 2016, 4, 7520-7536.

6 M. Samanta and K. Biswas, J. Am. Chem. Soc., 2017, 139, 9382-9391.

7 E. M. Levin, M. F. Besser and R. Hanus, J. Appl. Phys., 2013, 114, 083713.

8 P. A. Vermeulen, A. Kumar, G. H. ten Brink, G. R. Blake and B. J. Kooi, Cryst. Growth Des., 2016, 16, 5915-5922.

9 J. Davidow and Y. Gelbstein, J. Electron. Mater., 2012, 42, 1542-1549.

10 A. J. Thompson, J. W. Sharp and C. J. Rawn, J. Electron. Mater., 2009, 38, 1407-1411.

11 B. A. Cook, X. Wei, J. L. Harringa and M. J. Kramer, J. Mater. Sci., 2007, 42, 7643-7646.

12 B. A. Cook, M. J. Kramer, X. Wei, J. L. Harringa and E. M. Levin, J. Appl. Phys., 2007, 101, 053715.

13 S. H. Yang, T. J. Zhu, S. N. Zhang, J. J. Shen and X. B. Zhao, J. Electron. Mater., 2010, 39, 2127-2131.

14 E. M. Levin, B. A. Cook, J. L. Harringa, S. L. Bud'ko, R. Venkatasubramanian and K. Schmidt-Rohr, Adv. Funct. Mater., 2011, 21, 441-447.

15 E. M. Levin, S. L. Bud'ko and K. Schmidt-Rohr, Adv. Funct. Mater., 2012, 22, 2766-2774.

16 T. Zhu, H. Gao, Y. Chen and X. Zhao, J. Mater. Chem. A, 2014, 2, 3251-3256.

17 J. R. Salvador, J. Yang, X. Shi, H. Wang and A. A. Wereszczak, J. Solid State Chem., 2009, 182, 2088-2095.

18 A. Kumar, P. A. Vermeulen, B. J. Kooi, J. Rao, L. van Eijck, S. Schwarzmüller, O. Oeckler and G. R. Blake, Inorg. Chem., 2017, 56, 15091-15100.

19 Y. Chen, C. M. Jaworski, Y. B. Gao, H. Wang, T. J. Zhu, G. J. Snyder, J. P. Heremans and X. B. Zhao, New J. Phys., 2014, 16, 013057.

20 S. Perumal, S. Roychowdhury, D. S. Negi, R. Datta and K. Biswas, Chem. Mater., 2015, 27, 7171-7178.

21 A. C. Larson and R. B. von Dreele, Los Alamos National Laboratory LAUR Report, No. 86-748, 2004.

22 L. Dusza, High Temp.-High Pressures, 1995-1996, 27/28, 467473.

23 T. Schröder, T. Rosenthal, N. Giesbrecht, M. Nentwig, S. Maier, H. Wang, G. J. Snyder and O. Oeckler, Inorg. Chem., 2014, 53, 7722-7729.

24 H. Wang, W. D. Porter, H. Böttner, J. König, L. Chen, S. Bai, T. M. Tritt, A. Mayolet, J. Senawiratne, C. Smith, F. Harris, P. Gilbert, J. Sharp, J. Lo, H. Kleinke and L. Kiss, J. Electron. Mater., 2013, 42, 1073-1084.

25 P. W. Stephens, J. Appl. Crystallogr., 1999, 32, 281-289. 
26 M. N. Schneider, P. Urban, A. Leineweber, M. Döblinger and O. Oeckler, Phys. Rev. B, 2010, 81, 184102.

27 L. S. Ramsdell, Am. Mineral., 1947, 32, 64-82.

28 T. Siegrist, P. Merkelbach and M. Wuttig, Annu. Rev. Condens. Matter Phys., 2012, 3, 215-237.

29 T. Rosenthal, M. N. Schneider, C. Stiewe, M. Döblinger and O. Oeckler, Chem. Mater., 2011, 23, 4349-4356.

30 O. G. Karpinsky, L. E. Shelimova, M. A. Kretova and J. P. Fleurial, J. Alloys Compd., 1998, 268, 112-117.

31 F. Fahrnbauer, D. Souchay, G. Wagner and O. Oeckler, J. Am. Chem. Soc., 2015, 137, 12633-12638.

32 F. Fahrnbauer, P. Urban, S. Welzmiller, T. Schröder, T. Rosenthal and O. Oeckler, J. Solid State Chem., 2013, 208, 20-26.

33 T. Rosenthal, L. Neudert, P. Ganter, J. de Boor, C. Stiewe and O. Oeckler, J. Solid State Chem., 2014, 215, 231-240.

34 E. S. Božin, C. D. Malliakas, P. Souvatzis, T. Proffen, N. A. Spaldin, M. G. Kanatzidis and S. J. L. Billinge, Science, 2010, 330, 1660-1663.

35 P. Fons, A. V. Kolobov, M. Krbal, J. Tominaga, K. S. Andrikopoulos, S. N. Yannopoulos, G. A. Voyiatzis and T. Uruga, Phys. Rev. B, 2010, 82, 155209.
36 T. Matsunaga, P. Fons, A. V. Kolobov, J. Tominaga and N. Yamada, Appl. Phys. Lett., 2011, 99, 231907.

37 K. V. Mitrofanov, A. V. Kolobov, P. Fons, M. Krbal, T. Shintani, J. Tominaga and T. Uruga, Phys. Rev. B, 2014, 90, 134101.

38 K. R. Knox, E. S. Bozin, C. D. Malliakas, M. G. Kanatzidis and S. J. L. Billinge, Phys. Rev. B, 2014, 89, 014102.

39 S. Christensen, N. Bindzus, M. Sist, M. Takata and B. B. Iversen, Phys. Chem. Chem. Phys., 2016, 18, 1587415883.

40 M. Sist, H. Kasai, E. M. J. Hedegaard and B. B. Iversen, Phys. Rev. B, 2018, 97, 094116.

41 C. W. Li, J. Ma, H. B. Cao, A. F. May, D. L. Abernathy, G. Ehlers, C. Hoffmann, X. Wang, T. Hong, A. Huq, O. Gourdon and O. Delaire, Phys. Rev. B, 2014, 90, 214303.

42 M. Sist, E. M. J. Hedegaard, S. Christensen, N. Bindzus, K. F. F. Fischer, H. Kasai, K. Sugimoto and B. B. Iversen, IUCrJ, 2016, 3, 377-388.

43 T. Keiber, F. Bridges and B. C. Sales, Phys. Rev. B, 2013, 111, 095504. 